\title{
Treatment outcomes of severe acute malnutrition in children treated within Outpatient Therapeutic Program (OTP) at Wolaita Zone, Southern Ethiopia: retrospective cross-sectional study
}

Mulugeta Yohannis Kabalo ${ }^{1 *}$ and Canaan Negash Seifu ${ }^{2}$

\begin{abstract}
Background: Children in third world countries suffer from severe acute malnutrition (SAM) in an extent of public health important. SAM management protocol available this time brought the approach from facility-based to community-based by Outpatient Therapeutic Program (OTP). But, little was known about the treatment outcomes of the program in Ethiopia. Thus, this study was aimed to assess treatment outcomes of SAM and identify factors associated among children treated at OTP in Wolaita Zone.
\end{abstract}

Methods: A retrospective facility-based cross-sectional study was conducted in OTP records of 794 children, treated at 24 health posts retrieved from January to December 2014. Population proportion to size (PPS) was used to allocate sample for each selected district and OTP sites within district. Individual cards of children were selected by systematic random sampling. Data were entered, thoroughly cleaned, and analyzed in SPSS version 20.

Results: The recovery rate was revealed as $64.9 \%$ at $95 \% \mathrm{Cl}(61,68)$. Death rate, default rate, weight gain, and length of stay were $1.2 \%, 2.2 \%, 4.2 \mathrm{~g} / \mathrm{kg} /$ day, and 6.8 weeks respectively. Children living in $<25 \mathrm{~min}$ were with 1.53 times higher odds of recovery than children residing in $\geq 25 \mathrm{~min}(A O R=1.53$ at $95 \% \mathrm{Cl}(1.11,2.12))$. The likelihood of recovery was 2 . 6 times higher for children with kwashiorkor than for those with marasmus (AOR $=2.62$ at $95 \% \mathrm{Cl}(1.77,3.89)$ ). Likewise, children provided with amoxicillin were 1.52 times more likely to recover compared to their counterparts ( $A O R=1.52$ at $95 \% \mathrm{Cl}(1.09,2.11))$.

Conclusions: The recovery rate and weight gain were lower than sphere standard. Distance from OTP, provision of amoxicillin, and type of malnutrition were factors identified as significantly associated with treatment outcome of SAM. Building capacity of OTP service providers and regular monitoring of service provision based on the management protocol were recommended.

Keywords: Outpatient Therapeutic Program, Severe acute malnutrition, Treatment outcome

\footnotetext{
* Correspondence: yohannismulugeta@yahoo.com

${ }^{1}$ School of Public Health, Wolaita Sodo University, Southern Ethiopia, P.O.Box

126, Wolaita Sodo, Ethiopia

Full list of author information is available at the end of the article
} 


\section{Background}

Acute malnutrition is the short-term response to inadequate nutritional intake that often occurs in combination with infections [1]. It is classified as moderate acute malnutrition (MAM) and severe acute malnutrition (SAM) based on their severity $[1,2]$, explicitly indicated by children weight for height $Z$-score (WHZ) and/or weight for height/ length (WFH \%), presence of edema, and mid-upper arm circumference (MUAC). Acute malnutrition is defined as SAM when $\mathrm{WHZ}<-3$, MUAC $<115 \mathrm{~mm}$, and/or edema $+/++$ [2]. It contributes to 1.7 million child deaths per year in sub-Saharan Africa and carries nine times higher risk of death than that for a healthy child $[1,3]$.

The prevalence of SAM in children at developing countries was around 2\% [4]. According to the Ethiopian Demographic and Health Survey (EDHS) 2011, 3\% of under-five children were severely wasted. The same report in Southern Nations, Nationalities, and Peoples' Region (SNNPR) indicates the prevalence of severe wasting in under-five children as $1.9 \%$ [5]. Moreover, SAM in Wolaita Zone was as about $2.5 \%$ from different reports $[6,7]$. Thus, it is a public health problem in most developing world including Ethiopia.

Previously, this SAM was managed in health facilities and therapeutic feeding centers (TFC) with its own shortcomings $[8,9]$. Some of the challenges were limited coverage and impact, costliness, cross infections, and high mortality rate $[4,10]$. To reduce limitations, communitybased management of acute malnutrition (CMAM) was endorsed with joint statement of WHO and UNICEF in 2007 [10]. This advances decentralized management system to the community-based approach. Therefore, community outreach, outpatient management of SAM children without medical complications (OTP), inpatient management of SAM with medical complications (SC), and the program address MAM were designed as components of CMAM $[2,4,9]$.

OTP serve management of SAM in children aged 659 months [4]. Children will be admitted to the program when MUAC $<11.5 \mathrm{~cm}$, nutritional edema is $+/++$, they passed the appetite test, and no medical complications identified $[4,10]$. The management of program were mainly with ready-to-use therapeutic foods (RUTF); other routine medications like antibiotics, vitamin $\mathrm{A}$, and folic acid; and dewormings [2, 11]. Children obtain weekly RUTF based on their weight and routine supportive therapies based on their requirement according to SAM management protocol $[4,12]$.

Children admitted to OTP are discharged by their respective admission criteria [4]. That is, MUAC admittances are assessed for recovery or progress by MUAC and the same works if admitted by WFH\%. Nevertheless, admissions with only edema are discharged by anthropometric indicator like MUAC and WFH\% as well. Consequently, children were discharged as recovered when MUAC $\geq 12.5 \mathrm{~cm}$ and no edema for at least two consecutive weeks. However, consistently used discharge criteria to declare recovery is $15 \%$ weight gain from admission weight for children admitted with wasting and after disappearance of edema for two consecutive weeks for children admitted with edema [10, 13]. Children discharged from OTP are periodically monitored to avoid relapse [14].

The outcomes were declared as recovered, defaulted, died, medical transfer, and non-respondent for treatment, based on the management protocol $[4,10]$. The treatment outcomes were compared with international sphere standard requirements to evaluate the program effectiveness [4]. The recovery, death, and default rates were considered as acceptable when $>75,<10$, and $<15 \%$ respectively and alarming when $<50,>15$, and $>25 \%$ respectively based on international sphere standard [15]. Moreover, weight gain, length of stay, and coverage were thought as acceptable when $\geq 8 \mathrm{~g} / \mathrm{kg} /$ day, $<4$ weeks, and $>50-70 \%$, respectively, and considered as alarming when $<8 \mathrm{~g} / \mathrm{kg} /$ day, $>6$ weeks, and $<40 \%$ respectively $[4,10,15]$.

The performance of OTP for the management of SAM in children was less explored and factor associated with the performance of the program were also not significantly investigated $[11,16]$. Since the endorsement of the program, there were little studies done to elucidate the effectiveness of OTP in Ethiopia. Therefore, assessment of treatment outcomes of SAM in children treated at OTP and factors affecting the treatment outcomes require to be studied. Thus, this study fills the information gaps by determining treatment outcomes with SAM management performance indicators and identifying factors associated with treatment outcome among children treated at OTP in Wolaita Zone. That would add significant input for the program to set long-term planning for CMAM services in general and OTP in particular.

\section{Methods \\ Study setting}

The study was conducted in Wolaita Zone, SNNPRS, Ethiopia. Wolaita Zone (study area) is one of the zonal administrations in the southern region of Ethiopia, located $390 \mathrm{~km}$ south of Addis Ababa. Total population of this study area was estimated as $1,762,682$, where $51 \%$ were females and 274,978 were children under 5 years of age. The annual report of zonal health office indicated that 19,390 under-five SAM children were managed at OTP in 2014. The study area in general had 12 administrative districts and three town administrations. Likewise, this area share three hospitals, 70 health centers, and 380 health posts [17].

OTP was piloted in Ethiopia, Malawi, Sudan, and Niger in Africa, for its effectiveness on SAM management from 2000-2006 and endorsed in 2007. Thus, piloting was done 
from 2000 in the study area and the program was legitimated in 2007 [18-21]. Hence, starting from 2007, the program was implemented in the study area. The program had been implemented in all districts of Wolaita Zone, starting from the consent. However, majority of children managed in OTP exist in Boloso Sore, Damot Sore, Damot Gale, and Boloso Bombe districts. Thus, SAM children managed in OTP of Wolaita Zone at the indicated districts were 12,216 (63\%) in 2014 [17].

\section{Study design and period}

A retrospective facility-based cross-sectional study was conducted on records from January to December in 2014. The study period of this research work was from July to August in 2015 as of data collection period.

\section{Source and study population}

All children (6-59 months) admitted to OTP of Wolaita Zone, with a diagnosis of SAM in 2014, were source population. All children selected by systematic random sampling from randomly selected OTP sites within selected districts in the study area were study populations.

\section{Inclusion and exclusion criteria}

Records of children admitted to OTP from January to December 2014 were included. Children who are readmitted were excluded. Moreover, children whose sociodemographic variables and admission criteria are not registered in their OTP card were also excluded.

\section{Sample size determination and sampling procedure}

Sample size used for this study was calculated by means of the software Epi-info version 6. The assumptions considered were $95 \%$ confidence level, power $80 \%$, and relative risk of appetite test with plumpy'nut 4.5, from study conducted in Tigray Region, within treatment outcomes of SAM and their determinants in OTP [11]. Thus, the sample size determined was 794 children represented by their medical cards. Population proportion to size (PPS) was used to allocate the required sample size for each woreda and for each OTP site.

There are 12 districts in Wolaita administrative zone. From these districts, four were selected based on their high SAM case flow. The study area in average holds four health centers per district and five satellite health posts per health center in their catchment area. Therefore, in average, there are 20 health posts per district in Wolaita Zone [17]. Of those health posts (HP) or OTP sites, six sites per each district were selected by a simple random sampling technique. Then, a systematic random sampling technique was used to select individual child card (Fig. 1).

\section{Data collection procedures}

Structured and pretested data collection questionnaires were used to collect data from both registration books and children OTP record card. The records were reviewed mainly from children cards, and some variables missing in cards were checked from registration books. Data collectors were those who have $10+3$ educational level in nursing and are experienced in data collection. Trained data collectors did the record gathering from selected OTP sites and compiled the questionnaire.

\section{Study variables}

Dependent variable: treatment outcome

Independent variables: socio-demographic: Age, sex, distance to OTP and referral system

Variables related with admission and follow-up: type of malnutrition, physical examination, and history at admission, appetite test by RUTF, length of stay, date of minimum weight gain, RUTF, and other routine medications.

\section{Operational definitions}

Treatment outcome: grouped as recovered and not recovered from SAM management at OTP in this study

Recovered: children attained 15\% weight gain from admission weight for children admitted with wasting and after two consecutive weeks from edema disappeared for edematous children.

Not recovered: defined as children discharged from OTP with outcome other than recovery in this study (death, default, non-responder, medical transfer, and falsely recovered).

Type of malnutrition: grouped as marasmus (nonedematous), kwashiorkor (edematous), and marasmic kwashiorkor (both edema and severe wasting).

Distance to OTP site: indicates time taken from OTP site to house/residence of children by foot in hour/ minutes.

Adequacy of RUTF provision: sufficiency of RUTF was calculated based on the national SAM management protocol standing from weight of respective children.

\section{Data quality control and management}

Data were collected with close supervision at the time of collection. After proper collection, information were checked up by supervisors for completeness and consistency regularly until data collection is completed. Subsequent to fitting collection, data were carefully entered, cleaned, coded, and analyzed in SPSS version 20.

Systematic data cleaning procedures were carried out to assure data quality of both categorical and continuous variables [22]. Data were cleaned orderly by firstly sorting each variable in ascending order to check for unexpected cases. Variables identified as unexpected and extreme were verified with its respective checklist. Further cleaning 


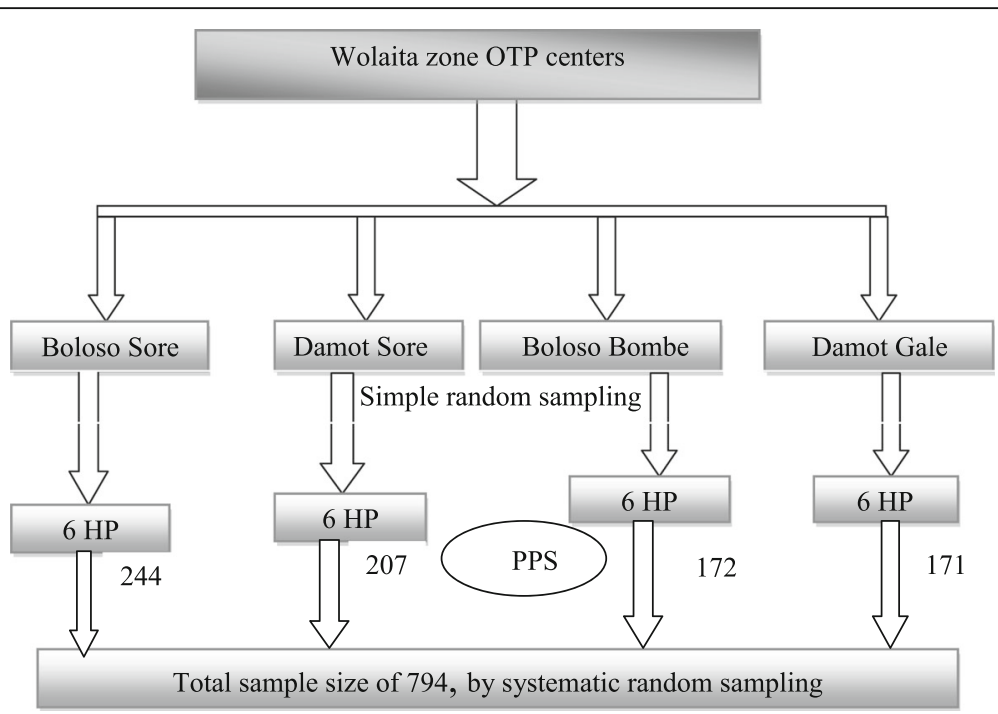

Fig. 1 Sampling procedure schematic presentation

was made by selecting randomly 80 (10\%) from the total number of participants.

\section{Data processing and analysis}

Data were analyzed by statistical summarization of exposure variables and description of the rates of treatment outcomes. The treatment outcomes were described by recovery, death, default, non-responders for treatment, and medical transfer rates. Both weight gain and length of stay were also calculated based on national protocol [4]. Later, bivariate logistic regression was used to analyze treatment outcome with exposure variables. Multivariable logistic regression was carried out to variables selected by binary regression ( $p$ value $<0.25$ ). Then, predictor variables of treatment outcome were reported by AOR at $95 \%$ confidence level (AOR at 95\% CI).

\section{Results}

Socio-demographic characteristics of children

The study included records of 794 children admitted to OTP from January to December 2014 in 24 health posts of four woredas in Wolaita Zone. Of children included, 422 (53.1\%) were female and 335 (42.2\%) were $<24$ months of age. The median age at admission was 24 months with IQR of 12 to 36 . Children referred to OTP mainly by community volunteers were 386 (65.8\%) and came from $<25 \mathrm{~min}$ of residence from OTP site to home on foot were 378 (53.8\%) (Table 1).

\section{Nutritional status and co morbidities at admission}

Children admitted to OTP based on edema were 273 (34.4\%) of the study participants. The rest were admitted based on MUAC. Hence, participants admitted to the program with MUAC $<11 \mathrm{~cm}$ were 475 (59.8\%), 11-11.5 cm were $28(3.5 \%)$, and $>11.5 \mathrm{~cm}$ were

Table 1 Socio-demographic characteristics of children admitted to OTP in Wolaita Zone in 2014

\begin{tabular}{|c|c|c|c|}
\hline Variables & Categories of variables & Frequencies & Percentage (\%) \\
\hline \multirow[t]{2}{*}{ Age } & $<24$ months & 335 & 42.2 \\
\hline & $\geq 24$ months & 459 & 57.8 \\
\hline \multirow[t]{2}{*}{ Sex } & Female & 422 & 53.1 \\
\hline & Male & 372 & 46.9 \\
\hline \multirow[t]{4}{*}{ Referral system } & Community volunteers & 386 & 65.8 \\
\hline & HEW & 90 & 15.3 \\
\hline & Self-referred & 68 & 11.6 \\
\hline & Other neighbors & 43 & 7.3 \\
\hline \multirow[t]{2}{*}{ Distance from OTP } & $\leq 24 \min$ & 378 & 53.8 \\
\hline & $\geq 25 \min$ & 324 & 46.2 \\
\hline
\end{tabular}


18(2.3\%). Of children with edema at admission, 149 (54.6\%), 111 (40.7\%), and 13 (4.8\%) had grades I, II, and III edema respectively. Moreover, the level of malnutrition was further classified as marasmus 503 (63.4\%), kwashiorkor 228 (28.7\%), marasmic kwashiorkor 45 (5.7\%), and MAM 18 (2.3\%) of children included. Children with one or more medical problems were 252 (31.7\%) in the study. Of comorbidities, $58(12.6 \%)$ had dehydration, 54 (6.8\%) had coughing, and 38 (4.8\%) had diarrhea at admission to mention some of the complications (Table 2).

\section{RUTF and routine medication provision}

RUTF provision is based on admission weight of children. In majority of the study participants, 562 (72.1\%) were adequately provided with RUTF in the program. More than half of children adequately supplied were female, 311 (55.3\%), and children with admission weight of $\leq 8 \mathrm{~kg}$ (smaller children), 300 (53.4\%). Non-edematous children were $372(66.2 \%)$ of participants sufficiently offered RUTF. Routine medications are also provided at OTP, but it was given for some children in the program within the study area. Of children provided medications, $356(44.8 \%)$ were given amoxicillin and $203(25.6 \%)$ were supplied with vitamin A (Table 2).

Table 2 SAM at admission and medications of children admitted to OTP in Wolaita Zone, 2014

\begin{tabular}{llll}
\hline Variables & $\begin{array}{l}\text { Categories of } \\
\text { variables }\end{array}$ & Frequencies & Percentage (\%) \\
\hline Type of & Marasmus & 503 & 63.4 \\
malnutrition & Kwashiorkor & 228 & 28.7 \\
& Marasmic & 45 & 5.7 \\
& kwashiorkor & & \\
& MAM & 18 & 2.3 \\
Adequacy of RUTF & Adequate & 562 & 72.1 \\
& Not adequate & 217 & 27.9 \\
Comorbidities & Present & 252 & 31.7 \\
& Absent & 542 & 68.3 \\
Appetite test & Passed & 776 & 97.7 \\
Breastfeeding & Failed & 18 & 3.3 \\
& Yes & 296 & 37.3 \\
Amoxicillin & No & 468 & 62.7 \\
& Medications given & 356 & 44.8 \\
Vitamin A & Not given & 438 & 55.2 \\
& Medications given & 203 & 25.6 \\
Folic acid & Not given & 591 & 74.4 \\
& Medications given & 119 & 15 \\
& Not given & 675 & 85 \\
\hline
\end{tabular}

Treatment outcomes and international sphere standard references

The performance indicators calculation and further analysis was made for 776 (97.7\%) children with SAM. Recovery rate deliberated based on sphere standard was 504 (64.9\%) (at 95\% CI $(61.5,68.1)$ ) from SAM children who participated in the study. The recovery was within $\leq 8$ weeks for $404(80.2 \%)$ of the recovered children. The rates of death, default, non-responder, medical transfer, and false recovery were 9 (1.2\%), 17 (2.2\%), 19 (2.4\%), 41 (5.3\%), and 186 (23.9\%), respectively, in this study. The mean (SD) length of stay at OTP was 6.8 (3.2) weeks, and children gain weight in average of $4.2 \mathrm{~g} / \mathrm{kg} /$ day for length of stay (Table 3 ).

Factors associated with treatment outcome of SAM at OTP Bivariate analyses were carried out to identify candidate variables for multivariable logistic regression. Variables with $p$ value $<0.25$ at binary logistic regression were selected as candidate for multivariable logistic regression. Thus, variables found associated with treatment outcome at multivariable logistic regression were distance from OTP to home, amoxicillin provision, and type of malnutrition. That is, children residing in $<25$ min were with 1.53 times higher odds of recovery than those residing in $\geq 25 \mathrm{~min}$ (AOR = 1.53 at $95 \%$ CI $(1.11,2.12)$ ). The odds of recovery was 2.6 times higher for children with kwashiorkor than for those with marasmus (AOR $=2.62$ at 95\% CI $(1.77,3.89)$ ). Children provided with amoxicillin were 1.52 times more likely to recover compared to their counterparts $(\mathrm{AOR}=1.52$ at 95\% CI $(1.09,2.11)$ ) (Table 4).

\section{Discussion}

The finding of this research mainly indicates treatment outcomes of OTP and factors identified as associated with treatment outcome of children treated from SAM. The rates of treatment outcomes were 504 (64.9\%), 186 (23.9\%), 9 (1.2\%), 17 (2.2\%), $4.2 \mathrm{~g} / \mathrm{kg} /$ days, and 6.8 weeks for recovery, false recovery, death, default rate, weight gain, and length of stay respectively. Factors identified as associated with treatment outcome were the distance from OTP to residence by foot, type of malnutrition, and amoxicillin provision in this study. Besides, the

Table 3 Performance indicators of OTP and sphere standard references, Wolaita Zone, 2014

\begin{tabular}{llll}
\hline $\begin{array}{l}\text { Performance } \\
\text { indicators }\end{array}$ & $\begin{array}{l}\text { Frequencies } \\
\text { of indicators }\end{array}$ & \begin{tabular}{l} 
International sphere standards \\
\cline { 2 - 4 }
\end{tabular} & Accerences \\
\hline Recovery rate & $504(64.9 \%)$ & $>75 \%$ & Alarming \\
Death rate & $9(1.2 \%)$ & $<10 \%$ & $<50 \%$ \\
Default rate & $17(2.2 \%)$ & $<15 \%$ & $>15 \%$ \\
Average weight gain & $4.2 \mathrm{~g} / \mathrm{kg} /$ days & $\geq 8 \mathrm{~g} / \mathrm{kg} /$ days & $<8 \mathrm{~g} / \mathrm{kg} /$ days \\
Length of stay & 6.8 weeks & $<4$ weeks & $>6$ weeks \\
\hline
\end{tabular}


Table 4 Bivariate and multivariable analysis of factors associated with treatment outcome of children in OTP, Wolaita Zone, 2014

\begin{tabular}{|c|c|c|c|c|c|}
\hline \multirow[t]{2}{*}{ Variables $(n)$} & \multirow[t]{2}{*}{ Categories } & \multicolumn{2}{|c|}{ Treatment outcome } & \multirow[t]{2}{*}{ OR $(95 \% \mathrm{Cl})$} & \multirow[t]{2}{*}{$\mathrm{AOR}(\% \mathrm{Cl})$} \\
\hline & & Recovered & Not recovered & & \\
\hline \multirow[t]{2}{*}{ Age (776) } & $<24$ months & 213 & 118 & $0.95(0.71,1.29)$ & \\
\hline & $\geq 24$ months & 291 & 154 & 1 & \\
\hline \multirow[t]{2}{*}{ Sex (776) } & Male & 243 & 119 & $1.19(0.89,1.61)$ & $1.17(0.85,1.62)$ \\
\hline & Female & 261 & 153 & 1 & 1 \\
\hline \multirow[t]{3}{*}{ Referral system (572) } & HEW & 66 & 23 & $1.66(0.99,2.77)$ & \\
\hline & Self-referred & 47 & 21 & $1.29(0.74,2.45)$ & \\
\hline & Volunteers & 263 & 152 & 1 & \\
\hline \multirow[t]{2}{*}{ Distance from OTP (685) } & $\leq 24 \min$ & 253 & 116 & $1.50(1.10,2.06)^{*}$ & $1.53(1.11,2.12)^{*}$ \\
\hline & $\geq 25 \mathrm{~min}$ & 187 & 129 & 1 & 1 \\
\hline \multirow[t]{3}{*}{ Type of malnutrition (776) } & Kwashiorkor & 178 & 50 & $2.47(1.72,3.54)^{* *}$ & $2.62(1.77,3.89)^{* *}$ \\
\hline & Marasmic kwashiorkor & 29 & 16 & $1.25(0.67,2.37)$ & $1.10(0.53,2.27)$ \\
\hline & Marasmus & 297 & 206 & 1 & 1 \\
\hline \multirow[t]{2}{*}{ Length of stay (776) } & $\leq 6$ weeks & 245 & 124 & $1.13(0.84,1.52)$ & \\
\hline & $>6$ weeks & 259 & 148 & 1 & \\
\hline \multirow[t]{2}{*}{ RUTF intake (761) } & Adequate & 364 & 184 & $1.14(0.82,1.59)$ & \\
\hline & Not adequate & 135 & 78 & 1 & \\
\hline \multirow[t]{2}{*}{ Diarrhea (776) } & Absent & 486 & 252 & $2.14(1.11,4.12)^{*}$ & $2.13(0.98,4.61)$ \\
\hline & Present & 18 & 20 & 1 & 1 \\
\hline \multirow[t]{2}{*}{ Breastfeeding (746) } & Yes & 188 & 103 & $0.97(0.71,1.32)$ & \\
\hline & No & 297 & 158 & 1 & \\
\hline \multirow[t]{2}{*}{ Amoxicilin provision (776) } & Yes & 242 & 111 & $1.34(0.99,1.80)$ & $1.52(1.09,2.11)^{*}$ \\
\hline & No & 262 & 161 & 1 & 1 \\
\hline \multirow[t]{2}{*}{ Vitamin A intake (776) } & Yes & 138 & 60 & $1.33(0.94,1.88)$ & $1.41(0.96,2.05)$ \\
\hline & No & 366 & 212 & 1 & 1 \\
\hline \multirow[t]{2}{*}{ Date of min.wt. gain (774) } & $\leq 3$ weeks & 446 & 233 & $1.22(0.78,1.89)$ & \\
\hline & $>3$ weeks & 58 & 37 & 1 & \\
\hline
\end{tabular}

${ }^{*} p<0.05 ;{ }^{* *} p<0.01$

incredibly decisive findings this study identified were gaps in reporting recovery rate of SAM in OTP.

Children admitted to the program by edema were 273(34.4\%) as specified elsewhere. It was notably large compared to the finding of similar study from Tigray Region (1.6\%) [11]. But, the finding was parallel with the report of a related study at Shebedido district (47.4\%) [23]. This indicates the admission report by edematous SAM varied by studies. The possible reason might be variation in sampling of children in older age group. That is, majority of children included in this study (57.8\%) were aged 2 years and above. Thus, it is credible that the level of edematous SAM is fairly higher at the indicated age group due to stress of stopping breastfeeding.

The result revealed 504 (64.9\%) SAM children admitted to OTP were recovered. This indicates the recovery rate was lower than sphere standard acceptable range [4]. The finding was also lower compared to 77.8 and $87 \%$ recovery rates from the study done in TFC of Jimma and southern region $[8,24]$. However, it was comparable to 61.8 and $67.7 \%$ recovery rates from study done in OTP of Tigray Region and Kamba district $[11,16]$. The disparities in reports from OTP and TFC might be due to dissimilarity in settings where SAM management was carried out.

Based on the finding, 186 (23.9\%) children managed in OTP were discharged having report as "recovered" without attaining discharge criteria of SAM management protocol. Hence, the report in the card of children indicates 690 (88.9\%) of SAM children included in the study recovered, while only $64.9 \%$ recovered based on the criteria. As per SAM management protocol, children admitted to OTP must be discharged as recovered after attaining 15\% weight gain from admission weight for wasted children. Edematous children discharged as cured should be after two successive weeks from edema departure $[2,4,21]$. The likely reason for false recovery reporting might be faulty use of RUTF, 
since it is sold as a commodity in the study area [25]. Besides, lack of ample awareness on existing SAM management at service providers is also an extra concern.

The recovery rate of SAM at OTP should be $>75 \%$ to say the program is clinically effective based on sphere standard [15]. But, the rate in this study was lower than the acceptable range of this standard. Beyond the protocol, children managed in the program have failed appetite test 18 (2.3\%), marasmic kwashiorkor 45 (5.7\%), and grade III edema 13 (1.6\%) of partakers. So, the lower recovery rate might be related with admission of children beyond SAM management protocol.

The average weight gain of children admitted with marasmus and recovered was $4.2 \mathrm{~g} / \mathrm{kg} /$ day being far out of acceptable range of international sphere standard [15]. The finding was slightly lower compared to reports of 5.23 and $5.76 \mathrm{~g} / \mathrm{kg} /$ day from Tigray Region and Kamba district $[11,16]$. The lower average weight gain beneath sphere standard and study findings may be explained by improper utilization of provided therapeutic foods and other supportive medications by children.

The death and default rates were found to be 9 $(1.2 \%)$ and $17(2.2 \%)$ in this study. Both indicators were in acceptable range of sphere standard [4]. Death rate was comparable with similar study findings from OTP $[11,16,26]$. But, default rate was lesser compared to that from studies in Kamba and Tigray but in line with study from Durame [11, 16, 26]. The relative lesser default rate in this study might be explained by decentralization of the program to health post in the study area.

Children residing in $<25$ min were 1.53 times more likely to recover than children living in $\geq 25 \mathrm{~min}$ (AOR = 1.53 at $95 \%$ CI $(1.11,2.12)$ ). The report offset with the study from Kamba district reported lack of significant association between recovery and residence of children [16]. Other study excluded residence from analysis due to poor registration in the program [11]. The finding of this study can be possibly explained by lack of consistent attendance of children residing in longer distance from OTP. Despite default rate was acceptable, children reside in longer distance were less likely to come OTP site regularly per week than living in near the site.

The odds of recovery was 2.6 times higher for children with kwashiorkor than for children with marasmus $(\mathrm{AOR}=2.62$ at $95 \% \mathrm{CI}(1.77,3.89))$. That agrees with reports from TFC in Ghana and OTP at sub-Saharan Africa $[3,27]$. But, it counters study in TFC in Jimma zone and clinical review of childhood SAM management $[28,29]$. Moreover, there is disparity of finding from Kamba district which reported absence of association in type of malnutrition and SAM recovery rate [16]. Children admitted with marasmus had dehydration 35 (60.3\%), vomiting 11 (78.6\%), and chest retraction $20(74.1 \%)$ in this study. Thus, the possible reason for lower recovery rate in marasmic children might be due to high comorbidities in marasmic children.

Children provided with amoxicillin were 1.52 times more likely to recover compared to their counterparts (AOR = 1.52 at $95 \%$ CI $(1.77,3.89))$. The finding was in line with the report from OTP in Tigray Region and clinical trial in antibiotics as part of SAM management in India $[11,30]$. The likely recovery of children provided with amoxicillin were explained by supportive effect of antibiotics mainly amoxicillin in treatment progress of SAM at OTP. The antibiotic provided for routine treatment must be active against small bowel bacterial overgrowth [4]. Thus, the finding possibly justified that amoxicillin was active against small bowel bacterial overgrowth in SAM children.

\section{Limitation of the study}

Presence of missing information for some variables because of using record and child OTP card as data source would be the possible limitation of this study.

\section{Conclusions}

The recovery rate and weight gain were lower than sphere standard acceptable ranges. On the other hand, death and default rates were within the range of standard in this study. Thus, OTP effectiveness was partial because recovery rate, length of stay, and weight gain were out of sphere standard. Factors identified as significantly associated with treatment outcome of SAM children treated in OTP were distance from OTP site, amoxicillin provision, and type of malnutrition. Moreover, the study leads to conclude as there were gaps in appropriate follow-up of SAM management protocol in the program at the study area. Therefore, stakeholders should manage to shape service providers of OTP with SAM management protocol. To increase the effectiveness of OTP, the performance should be regularly monitored. Special attention was also needed to build capacity of service providers because of current decentralization of OTP to health post. To reveal appropriate utilization of RUTF at home level, further study should be conducted. Besides, coverage of the program also needs to be studied to establish the met need.

\footnotetext{
Abbreviations

CMAM: Community-based management of acute malnutrition; NORAD: Norwegian Agency for Development; OTP: Outpatient Therapeutic Program; RUTF: Ready-to-use therapeutic food; SAM: Severe acute malnutrition

\section{Acknowledgements}

The authors would like to express their deepest acknowledgement to the NORAD project for their financial support in the progress of the research work. Furthermore, the authors would like to extend special gratitude to the data collectors and supervisors of this research for their sincere concern. Our leading thanks should go to Migbaru Yohannes (Guni), Gideon Baaza, Melkamu Yohannes (Enu), Etaferahu Elias, Nigatu Baaza Gidebo, Gashaw Ganebo, Bereket Yohannes, Elisabeth Badie (Etiye), and Yohannes Kabalo Shamanaa (Gaashee) for their inspired bear all throughout the evolution of this investigation endeavor.
} 


\section{Funding}

This study was sponsored by NORAD, but they had no role in the work of the research.

\section{Availability of data and materials}

The datasets analyzed during the current study were available from the corresponding author on reasonable request.

\section{Authors' contributions}

MYK had the principal role in the outset of ideas, development of the methods, data processing or analysis, interpretation of the findings, and writing of the whole article. CNS was the guide in the commencement of the research scheme, development of the design, and interpretation of the study findings. Both authors exhaustively read and approved the final version of the manuscript.

\section{Competing interests}

The authors declare that they have no competing interests.

\section{Consent for publication}

Not applicable.

\section{Ethics approval and consent to participate}

Ethical clearance was obtained from the ethical review committee of the College of Health Sciences of Wolaita Sodo University. Letter of cooperation was obtained from Wolaita Zone health office for each selected district, and each respective woreda gave a permission letter to access data from selected OTP sites. A unique SAM number was used to access child card.

\section{Publisher's Note}

Springer Nature remains neutral with regard to jurisdictional claims in published maps and institutional affiliations.

\section{Author details}

${ }^{1}$ School of Public Health, Wolaita Sodo University, Southern Ethiopia, P.O.Box 126, Wolaita Sodo, Ethiopia. ${ }^{2}$ School of Public Health, Wolaita Sodo University, Southern Ethiopia, P.O.Box 138, Wolaita Sodo, Ethiopia.

\section{Received: 14 April 2016 Accepted: 2 March 2017}

Published online: 09 March 2017

\section{References}

1. Hobbs B, Bush A. Acute malnutrition: an everyday emergency; a 10-point plan for tackling acute malnutrition in under-fives. In: Generation nutrition campaign report. 2014.

2. HTP. Techinical notes on management of severe acute malnutrition. In: Module 13. 2011.

3. Sadler K. Community-based therapeutic care: treating severe acute malnutrition in sub-Saharan Africa. London: University College London; 2010.

4. Golden M, Grellety Y. Protocol for the management of severe acute malnutrition. Ethiopia: $\mathrm{MOH} ; 2007$.

5. Central Stastical Agency (Ethiopia), ICF International. Ethiopian Demographic and Health Survey 2011. In: Central Statistical Agency, editor. Addis Ababa, Maryland: Central Statistical Agency and ICF International; 2012.

6. ENCU, EWRD. Emergency nutrition quarterly bulletin,(fourth quarter 2008). In: Emergency nutrition coordination unit early warning \& response department (disaster management \& food security sector). 2008.

7. ENCU, DPPA. Emergency nutrition, quarterly bulletin (second quarter 2008). In: Emergency Nutrition Coordination Unit Early Warning Department (Disaster Prevention and Preparedness Agency). 2008.

8. Teferi E, Lera M, Sita S, Bogale Z, Datiko DG, Yassin MA. Treatment outcome of children with severe acute malnutrition admitted to therapeutic feeding centers in Southern Region of Ethiopia. Ethiop J Health Dev. 2010;24(3).

9. UNICEF. Evaluation of community management of acute malnutrition (CMAM). Ethiopia: Federal Ministry of Health, Government of Ethiopia and UNICEF Country Office; 2012

10. WHO, UNICEF. Community-based management of severe acute malnutrition: a joint statement by the World Health Organization, the World Food Programme, the United Nations System Standing Committee on nutrition and UNICEF. 2007.
11. Henock GY, Carl K, Daniel N, Lemma W. Outpatient Therapeutic Feeding Program Outcomes and Determinants in Treatment of Severe Acute Malnutrition in Tigray, Northern Ethiopia: A Retrospective Cohort Study. Plos one. 2013;8(6).

12. $\mathrm{MOH}$. Integrated management of acute malnutrition guidelines. Republic of Uganda: Ministry of Health; 2010.

13. WHO. Updates on the management of severe acute malnutrition in infants and children. 2013.

14. Collins LS. Therapeutic nutrition for children with severe acute malnutrition: summary of African experience. Indian Pediatrics. 2010;47.

15. Charter H. Minimum standards in food security and nutrition. In: sphere hand book. 2011.

16. Shanka NA, Lemma S, Abyu DM: Recovery rate and determinants in treatment of children with severe acute malnutrition using outpatient therapeutic feeding program in Kamba District, South West Ethiopia. Nutr Disord Ther. 2015;5(2)

17. Departement H. Wolaita Zone Health Department, annual report. 2014.

18. FANTA, USAID. Review of community-based management of acute malnutrition in the post-emergency context: synthesis of lessons on integration of CMAM into national health systems. 2008.

19. Collins S, Sadler K, Dent N, Khara T, Guerrero S, Myatt M, Saboya M, Walsh A Key issues in the success of community-basedmanagement of severe malnutrition. In: Technical background paper. 2008.

20. Hedwig D, Anne S, Grant F, Navarro-Colorado C. Review of communitybased management of acute malnutrition (CMAM) in the post-emergency context: synthesis of lessons on integration of CMAM into national health systems Ethiopia, Malawi and Niger. 2008.

21. UNICEF, WHO. WHO child growth standards and the identification of severe acute malnutrition in infants and children: joint statement by the World Health Organization and the United Nations Children's Fund, vol. 20. Swezer land: WHO; 2009

22. Unwin A. SPSS survival manual: a step by step guide to data analysis using SPSS for Windows (Version 12). In: Pallant J, editor. Australia; 2005.

23. Merid M, Mengesha N, Balewgizie D, Sileshi T, Dessie Y. Treatment outcome and factors affecting time to recovery in children with severe acute malnutrition treated at outpatient therapeutic care program. Global Health Action. 2016:9.

24. Jarso H, Workicho A, Alemseged F: Survival status and predictors of mortality in severely malnourished children admitted to Jimma University Specialized Hospital from 2010 to. Jimma, Ethiopia: a retrospective longitudinal study. BMC Pediatrics. 2012;2015:15(76).

25. Tadesse E, Ekström EC, Berhane Y. Challenges in implementing the integrated community-based outpatient therapeutic program for severely malnourished children in rural Southern Ethiopia. Nutrients. 2016:8:251.

26. Boltena SS, Swart R. Factors affecting the rehabilitation outcome of children with severe acute malnutrition in Durame, Southern Ethiopia. Cape: University of the Western Cape; 2008.

27. Saaka M, Osman SM, Amponsem A, Ziem JB, Abdul-Mumin A, Akanbong P, Yirkyio E, Yakubu E, Ervin S. Treatment outcome of severe acute malnutrition cases at the tamale teaching hospital. Journal of Nutrition and Metabolism. 2015

28. Misganaw C, Mesfin M, Tesfaye M, Derese A. Retrospective study on outcome of in-patient treatment of severe acute malnutrition in Jimma University specialized hospital from September 2011-September 2012. Journal of Diagnostics. 2014;1(2):18-28.

29. Manary MJ, Sandige HL. Management of acute moderate and severe childhood malnutrition. BMJ. 2008:337:a2180.

30. Indi T, Hayley S, Goldbach SB, Lacey N, Guthrie J, Meuli BS, Richard J, Wang MD, Kenneth M, Maleta MB, Mark J. Antibiotics as Part of the Management of Severe Acute Malnutrition. N Engl J Med. 2013;368(5). 